“(C) 2018 IEEE. Personal use of this material is permitted. Permission from IEEE must be obtained for all other uses, in any current or future media, including reprinting/republishing this material for advertising or promotional purposes, creating new collective works, for resale or redistribution to servers or lists, or reuse of any copyrighted component of this work in other works." 


\title{
Frequency-Shaped Second-Order Sliding Mode Control for Smart Suspension Systems
}

\author{
Sayed Royel, Quang Ha and Ricardo P. Aguilera \\ School of Electrical and Data Engineering \\ University of Technology Sydney, Ultimo, NSW 2007, Australia \\ Sayed.Royel@uts.edu.au, Quang.Ha@uts.edu.au, Ricardo.Aguilera@uts.edu.au
}

\begin{abstract}
Design of a frequency-shaped second-order sliding mode (FS2SM) controller is demonstrated by means of exploiting second-order low-pass filter (LPF) to model the dynamic sliding surface to shape the frequency characteristics of the equivalent dynamics. The proposed technique is numerically verified in the simulation of a half-car model (HCM) with inbuilt active hydraulically interconnected suspension (HIS) system. The closedloop performances confirm that inclusion of an appropriate filter in the control scheme allows not only to reduce the roll angle but also its spectrum can be shaped.
\end{abstract}

\section{INTRODUCTION}

Autonomous driverless cars and unmanned ground vehicles (UGVs) are becoming a reality, thanks to the robotics community. These vehicles can be used in complex envrionments and off-road navigation, and are subject to nonlinear dynamic forces and moments because of complex terrain behavior and uneven traversing surface [1]-[3]. Due to unstructured terrain irregularities and size of the wheeled off-road vehicles, they can be considered among the primary sources of energy dissipation and pollutant emission; they should overcome as well as their performing tasks. The active hydraulicallyinterconnected suspension (HIS) is a promising candidate for rollover prevention, which is one of the prominent priorities in vehicle safety and handling control. Due to a turning maneuver, when the car body inclines, a differential pressure results in the HIS system which produces a counter roll moment to oppose that of the centrifugal force.

Sliding mode (SM) control (SMC) is known as a discontinuous robust control [4]-[6], which forcibly confines the system's states to a specified and user-chosen surface (is called sliding or switching surface) by oscillating the control structure with infinite frequency on both sides of a stable hyperplane in the state space. Hence, the ultimate trajectory does not exist entirely within one control structure, instead it slides along the boundaries of the control structures. The motion of the system as it slides along these boundaries is called a SM and the geometrical locus consisting of the boundaries is called the sliding (hyper) surface. SMC alters a possibly higher-order problem into a first-order stabilization problem that of controlling the distance from the system state to the manifold so that the distance is zero or as close as to that the actuators can obtain. The striking feature of SMC is its insensitivity to parametric uncertainty and disturbances during the SM [4],[7],[8].

At first, a sliding surface is designed such that the reduced order model has convergence properties and independent of the model uncertainty. The $n$th order sliding manifold can be defined as the static intersection in the state space by $\sigma(x)=$ $\mathcal{S} x=x_{n}+\sum_{k=1}^{n-1} \rho_{k} x_{k}=0, x \in \mathbb{R}^{n}, \mathcal{S}=\left[\rho_{1}, \cdots, \rho_{n-1}, 1\right]$, in which the parameters $\rho_{1}$ to $\rho_{n-1}$ are chosen such that the characteristic equation $s^{n-1}+\sum_{k=1}^{n-1} \rho_{k} s^{k-1}=0$ is Hurwitz, where $s$ is the Laplace operator [7], [9]. Secondly, the control gain is constructed (can be obtained based on the generalized Lyapunov stability theory), which can use only the information on the bounds of uncertain variables, to drive all trajectories on to the manifold in finite time and remain on it thereafter. The average of the high-frequency switching induces the control which ensures the conditon $\dot{\sigma}=0$ [7][10]. Since the polynomial is Hurwitz, once on the sliding manifold, the trajectories will go to zero with a transient behavior characterized by the selected $\rho_{1}, \ldots, \rho_{n-1}$.

The $r$-th order sliding mode $(r$-SM) can be determined by the equalities

$$
\sigma(x)=\dot{\sigma}(x)=\cdots=\sigma^{(r-1)}(x)=0, r=r_{1}, \ldots, r_{n},
$$

in which $(i)$ successive total time derivatives $\sigma, \ldots, \sigma^{(r-1)}$ are continuous functions of $x$, (ii) $\sigma=\cdots=\sigma^{(r-1)}=0$ is a nonempty integral set which consists locally of Filippov's trajectories [11], and (iii) the Filippov set of admissible velocities at the $r$-sliding points contains more than one vector [12]-[17]. The $r$-th derivative $\sigma_{1}^{\left(r_{1}\right)}, \sigma_{2}^{\left(r_{2}\right)}, \ldots, \sigma_{n}^{\left(r_{n}\right)}$ are discontinuous or non existent as a single-valued function of $x$ due to some reason like trajectory nonuniqueness. The vector $r=\left[r_{1}, \ldots, r_{n}\right]$ is called the sliding order. A SM is called stable if the corresponding integral sliding set is stable. The motion on $r$-sliding set (1) is said to be the 2-SM when the sliding order is $r=[2, \ldots, 2]$ with $\ddot{\sigma}$ are discontinuous and $\sigma, \dot{\sigma}$ are continuous functions of $x$.

Theoretically, the trajectory is supposed to slide on the sliding manifold, but there are delays and imperfections in the switching devices, and unmodeled high-frequency dynamics, which lead to chattering. Chattering is a small amplitude highfrequency oscillation which appears in the neighborhood of the sliding manifold that results in low control accuracy, high heat losses in electrical power systems, and high wear and tear of moving mechanical parts [4],[18]. It may excite unmodeled high-frequency dynamics which can also degrade the system performance and lead to unforeseen instabilities. Hence, it is one of the main concerns in SMC.

Frequency domain approach to structural control allows one to rolloff the control action at high frequencies and to specify 
the disturbance attenuation over desired bands of frequencies during control design. To extend the SMC design to the frequency domain, frequency-shaped (FS) SMC (FSSMC) and discrete-time FSSMC have been developed and applied to various mechanical systems including active vibration control [9], flexible robot manipulators [19],[20], active suspension system [21], and smart structure [22], [23]. In FSSMC, the sliding surface is modeled by a desired linear operator to suppress frequency components of the SM response in a designated frequency band [18], [19]. According to [19], linear operators can be interpreted as a low-pass filter (LPF) (either as a prefilter, similar to introducing artificial actuator dynamics or as a postfilter, functioning like sensor dynamics) for shaping the system equivalence dynamics in the frequency domain. A synthesis method for flexible manipulator was introduced in [20]: $(i)$ the terminal SMC was applied to achieve small steady state error and to accelerate the convergence of the sliding mode towards equilibrium and (ii) the FS approach was adopted to reduce the intrinsic resonance modes. A discrete time approach to the frequency shaping LQ control using the Parseval's theorem was studied for active suspension system in [21]. In [22], a FSSMC using output feedback was proposed for damping out the amplitude of vibration of smart flexible cantilever beam in which the system states are estimated based on measuring the output at a faster rate than the control input.

Notation and Definitions: The closed ball of radius $p$ with center at the origin is defined by $\mathcal{B}_{p} \triangleq\left\{x \in \mathbb{R}^{n}:\|x\| \leq p\right\}$; $p$ is a nonnegative real number. Let $r \in \mathbb{N}$ represents the sliding order. $\mathbb{R}$ and $\mathbb{N}$ denote the real and nonnegative natural number sets. $\lambda(\mathcal{A})$ is the spectrum of the non-defective matrix A. $\mathcal{A}^{\mathrm{T}}(x)^{\mathrm{T}}$ is the transpose of a matrix $\mathcal{A}$ (a vector $x$ ). $\mathcal{A}^{-1}(f)^{-1}$ is the inverse of a nonsingular square matrix $\mathcal{A}$ (a function $f$ ). $\mathbf{I}$ and $\mathbf{0}$ denote the identity and zero matrices of appropriate dimension. $\|\cdot\|_{1}$ denotes 1 -norm. $\operatorname{sgn}(\cdot), \operatorname{sat}(\cdot / \varepsilon)$ and $\tanh (\cdot / \varepsilon)$ denote respectively the sign function, high-slope saturation function and hyperbolic tangent function whose steepness is determined by $\varepsilon$ (is a small positive constant).

\section{CONTROL DeSign}

We consider the two-dimensional, single-input nonlinear system to illustrate the design methodology:

$$
\dot{x}_{1}=x_{2}, \quad \dot{x}_{2}=h(x)+g(x) u,
$$

where $h(x)$ and $g(x) \geq g_{0}>0, \forall x \in \mathbb{R}^{2}$, are some smooth nonlinear functions, and $u \in \mathbb{R}$ is the control input. Our main goal is to design a robustly stabilizing control law. In other words, the control $u=U\left(x_{1}, x_{2}\right)$ is supposed to constrains the trajectories to the manifold (or surface) $\sigma \equiv 0$ in finite time, i.e. $\lim _{t \rightarrow \infty} x_{1}, x_{2}=0$.

The standard ( $\dot{\sigma}$ is discontinuous and $\sigma$ is continuous functions of $x$ ) sliding variable can be expressed by a firstorder form $\sigma=\mathcal{S} x=\rho x_{1}+x_{2}, \mathcal{S}=[\rho 1], t \geq 0$. Here, $\rho$ satisfies the Hurwitz condition, i.e. $\rho>0$. Suppose that sliding motion is generated in the system at $t_{s}>0$. Hence, on this manifold $\sigma \equiv 0$, we have the governing motion $\dot{x}_{1}+\rho x_{1}=$ $0, \forall t \geq t_{s}>0$ and a solution $x_{1}(t)=x_{1}\left(t_{s}\right) e^{-\rho\left(t-t_{s}\right)}$ and its derivative $x_{2}(t)=\dot{x}_{1}(t)=-\rho x_{1}\left(t_{s}\right) e^{-\rho\left(t-t_{s}\right)}$, in which the rate of convergence can be controlled by choice of a finite positive constant $\rho$. For example, if the $k$-th stiffness and damping of an $n$ degree-of-freedom (dof) structure are known, and denoted respectively by $k_{k}$ and $c_{k}$, then we can select $\rho_{k}=k_{k} / c_{k}$ where $k=1,2, \cdots, n$.

In order to dynamically shape the frequency response of the equivalent dynamics, the sliding function is cast by using a dynamic linear operator $L(s)$, a function of $s$ [9], [23], [25], instead of $\rho$. We herein design the dynamic sliding surface (is a type of dynamic manifold) with a second-order LPF by

$$
\sigma=\mathcal{L}(s) x=\frac{b_{0}}{s^{2}+a_{1} s+a_{0}} x_{1}+x_{2}, \mathcal{L}(s)=[L(s) 1],
$$

for example, to get a steeper rolloff of $|L(j \omega)|, \omega \in[0, \infty)$ for large values of frequency $\omega$. Herein, $L(s)=\frac{b_{0}}{s^{2}+a_{1} s+a_{0}}$ gives a $|L(j \omega)|$ with 2-pole rolloff (an asymptotic slope of $-40 \mathrm{~dB} /$ decade above cut-off frequency) at high frequencies.

The algebraic manipulation of (3) gives

$$
\begin{aligned}
& \ddot{\sigma}+\sigma\left(a_{1} s+a_{0}\right)=\ddot{x}_{2}+x_{2}\left(a_{1} s+a_{0}\right)+b_{0} x_{1} \\
\Rightarrow & \ddot{\sigma}=\ddot{x}_{2}-\left(a_{1} s+a_{0}\right)\left(\frac{b_{0}}{s^{2}+a_{1} s+a_{0}}\right) x_{1}+b_{0} x_{1} \\
\Rightarrow \ddot{\sigma}= & \frac{\partial h}{\partial x}(h+g u)+\frac{\partial g}{\partial x}(h+g u) u+ \\
& g(x) \dot{u}+b_{0} x_{1}\left(1-\frac{a_{1} s+a_{0}}{s^{2}+a_{1} s+a_{0}}\right) \\
\Rightarrow & \ddot{\sigma}=H(x, u)+g(x) v+b_{0} x_{1}\left(\frac{s^{2}}{s^{2}+a_{1} s+a_{0}}\right) \\
\Rightarrow \ddot{\sigma}= & H(x, u)+s^{2} L(s) x_{1}+g(x) v,
\end{aligned}
$$

where $H(x, u)=\frac{\partial h}{\partial x}(h+g u)+\frac{\partial g}{\partial x}(h+g u) u$ and $v=\dot{u}$ is prescribed as the new control [26].

Let $\hat{g}$ and $\hat{H}$ be nominal model of the $g$ and $H$, respectively. We can obtain the best approximation $\hat{v}$ of the continuous control law that achieves $\ddot{\sigma}=0$ :

$$
\hat{v}=-\hat{g}(x)^{-1}\left[\hat{H}+s^{2} L(s) x_{1}\right] .
$$

A discontinuous term now can be added to pull the system to the surface as equivalent control $v_{E}=\hat{v}$ is valid only on the sliding surface. Adding a reaching control input $v_{R}$ to (5) guarantees that the plant dynamics reach the sliding surface in finite time regardless the presence of uncertainty or nonlinearity.

Substituting $v=v_{E}+v_{R}$ into (4) results in

$$
\begin{aligned}
\ddot{\sigma} & =H+s^{2} L(s) x_{1}+g(x)\left[-\frac{\hat{H}+s^{2} L(s) x_{1}}{\hat{g}(x)}+v_{R}\right] \\
& =H-\frac{g(x)}{\hat{g}(x)} \hat{H}+s^{2} L(s) x_{1}\left[1-\frac{g(x)}{\hat{g}(x)}\right]+g(x) v_{R} \\
& =\delta(x)+g(x) v_{R},
\end{aligned}
$$

where $\delta(x)$ denotes the perturbation term by

$$
\delta(x):=H-\frac{g(x)}{\hat{g}(x)} \hat{H}+s^{2} L(s) x_{1}\left[1-\frac{g(x)}{\hat{g}(x)}\right] .
$$

Suppose $\delta(x)$ satisfies the inequality

$$
\left|g(x)^{-1} \delta(x)\right| \leq \varrho(x)
$$

for some known function $\varrho(x)$. 
We consider a Lyapunov function candidate $V=\frac{1}{2} \dot{\sigma}^{2}$ for $\ddot{\sigma}=H(x, u)+s^{2} L(s) x_{1}+g(x) v$. Taking into account the inequality (7), the time derivative of $V$ can be computed as

$$
\begin{aligned}
\dot{V} & =\dot{\sigma} \ddot{\sigma}=\dot{\sigma}\left[\delta(x)+g(x) v_{R}\right] \\
& \leq g(x)|\dot{\sigma}| \varrho(x)+g(x) \dot{\sigma} v_{R} .
\end{aligned}
$$

To achieve our goal, the robust signal $v_{R}$ is taken as

$$
v_{R}=-\beta(x) \operatorname{sgn}(\dot{\sigma})-\kappa \dot{\sigma}, \kappa>0,
$$

with

$$
\beta(x) \geq \varrho(x)+\eta, \eta>0,
$$

so that the term $g(x) \dot{\sigma} v_{R}$ is negative and dominates over the residual (positive) term $g(x)|\dot{\sigma}| \varrho(x)$ when $\dot{\sigma} \neq 0$, and the net results (negative) to force $|\dot{\sigma}|$ to reach zero. Finally, the control structure is obtained as

$$
v=-\hat{g}(x)^{-1}\left[\hat{H}+s^{2} L(s) x_{1}\right]-\beta(x) \operatorname{sgn}(\dot{\sigma})-\kappa \dot{\sigma} .
$$

Substituting (9) into (8) results in

$$
\begin{aligned}
\dot{V} & \leq g(x)|\dot{\sigma}| \varrho(x)-g(x) \beta(x) \dot{\sigma} \operatorname{sgn}(\dot{\sigma})-g(x) \kappa \dot{\sigma}^{2} \\
& \leq g(x)|\dot{\sigma}| \varrho(x)-g(x)(\varrho(x)+\eta)|\dot{\sigma}|-g(x) \kappa \dot{\sigma}^{2} \\
& \leq-g(x) \eta|\dot{\sigma}|-g(x) \kappa \dot{\sigma}^{2} \leq-g_{0} \eta|\dot{\sigma}|-g_{0} \kappa \dot{\sigma}^{2} \\
& \leq-g_{0} \kappa \dot{\sigma}^{2}=-2 g_{0} \kappa V,
\end{aligned}
$$

where $g_{0}$ is defined in (2). By separating the variables and integrating the differential inequality over the time interval $t_{0} \leq \tau \leq t$, we obtain

$$
V(t) \leq V\left(t_{0}\right) e^{-2 \kappa g_{0}\left(t-t_{0}\right)} .
$$

According to the above inequality, we see that $V(t)$ will tend to zero exponentially in which the exponential decay rate is determined by the parameter $\kappa$. In (12), we established

$$
\dot{\sigma} \ddot{\sigma} \leq-g_{0} \eta|\dot{\sigma}|-g_{0} \kappa \dot{\sigma}^{2} .
$$

Since $g_{0} \kappa \dot{\sigma}^{2} \geq 0$, an $\eta$-reachability condition has been established and a sliding motion will take place. By neglecting the nonlinear term in (14) yields

$$
\frac{d}{d t}|\dot{\sigma}(t)| \leq-g_{0} \kappa|\dot{\sigma}(t)|,
$$

which implies that

$$
|\dot{\sigma}(t)| \leq\left|\dot{\sigma}\left(t_{0}\right)\right| e^{-\kappa g_{0}\left(t-t_{0}\right)} .
$$

Here, $\left|\dot{\sigma}\left(t_{0}\right)\right|$ represents the initial distance away from the sliding surface, and $\kappa$ is the decay rate at which the sliding surface is attained.

During the sliding phase, the motion is completely independent of $g$ and $H$, and we only need the information of the upper bound $\varrho(x)$ which is likely to be smaller than an upper bound on the whole function. As a result, the amplitude of the switching component, $\varrho(x)+\eta$ to $-\varrho(x)-\eta$, will be reduced. The parameters $\eta$ and $\kappa$ are positive tuning parameters to be chosen to obtain a desired closed-loop performance.

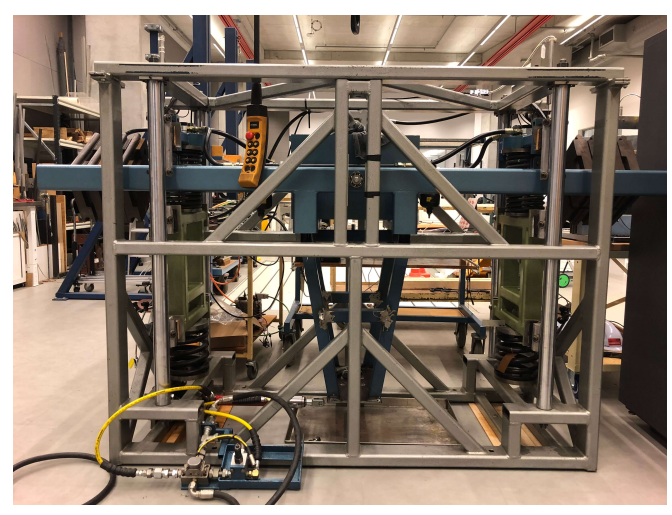

(a) Front view photograph

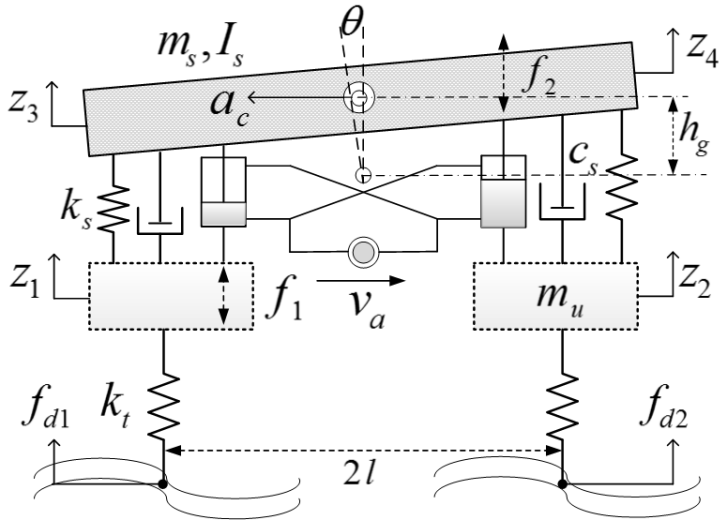

(b) Schematic of the HCM integrated with HIS system

Fig. 1: Input and output variables are, respectively, $u=v_{a}$ and $y=\left[z_{1}, z_{2}, z_{s}, \theta\right]^{\mathrm{T}}$. $v_{a}$ is the hydraulic fluid volumetric displacement produced by the actuator. $z_{1}, z_{2}, z_{3}, z_{4}$ denote left and right deflections of the tire and sprung mass, respectively. $z_{s}=\frac{z_{3}+z_{4}}{2}$ is the body bounce. $\theta=\frac{z_{4}-z_{3}}{2 l}$ is the roll angle.

\section{Simulation StUdy}

The proposed technique is numerically verified on a fourdof half-car model (HCM) (which simulates vehicle lateral dynamics and roll angle response to lateral acceleration) with an inbuilt active hydraulically-interconnected suspension (HIS) system. The HCM parameters are identified as follows: the vehicle body (sprung) mass, wheel (unsprung) mass and rotational inertia are $m_{s}=475 \mathrm{~kg}, m_{u}=35 \mathrm{~kg}$ and $I_{s}=120$ $\mathrm{kgm}^{2}$, respectively; the suspesion spring and tire stiffness are $k_{s}=20$ and $k_{t}=172 \mathrm{kN} / \mathrm{m}$, respectively, and the suspesion damping is $c_{s}=240 \mathrm{Ns} / \mathrm{m}$. The distance between the roll center and the center of gravity (CoG) is $h_{g}=0.5 \mathrm{~m}$, and the wheel track width is $2 l=0.7 \mathrm{~m}$. The active HIS system parameters are adopted from [24]. A photograph and schematic representation of the integrated system, available at the University of Technology Sydney (UTS) Laboratory, are shown in Figs. 11(a) and (b), respectively.

Using the Lagrange principle or Newton's law, we can describe the equations of motion in matrix form as

$$
M \ddot{z}+C \dot{z}+K z=B_{u} u+W_{d} f_{d},
$$

where $z=\left[z_{1}, z_{2}, z_{3}, z_{4}\right]^{\mathrm{T}}, u=\left[f_{1}, f_{2}\right]^{\mathrm{T}}, f_{d}=\left[f_{d 1}, f_{d 2}, a_{c}\right]^{\mathrm{T}}$. $f_{1}, f_{2}$ represent the forces produced by the active HIS. Two 
road disturbance inputs and the centrifugal acceleration are denoted by $f_{d 1}, f_{d 2}$ and $a_{c}$, respectively. The inertia, damping, stiffness, active HIS input and the disturbances input are, respectively, given by

$$
\begin{aligned}
M= & {\left[\begin{array}{cccc}
35 & 0 & 0 & 0 \\
0 & 35 & 0 & 0 \\
0 & 0 & 179.97 & 57.53 \\
0 & 0 & 57.53 & 179.97
\end{array}\right] \mathrm{kg}, } \\
C= & {\left[\begin{array}{cccc}
240 & 0 & -240 & 0 \\
0 & 240 & 0 & -240 \\
-240 & 0 & 240 & 0 \\
0 & -240 & 0 & 240
\end{array}\right] \mathrm{Ns} / \mathrm{m}, } \\
K= & {\left[\begin{array}{ccccc}
192000 & 0 & -20000 & 0 \\
0 & 192000 & 0 & -20000 \\
-20000 & 0 & 20000 & 0 \\
0 & -20000 & 0 & 20000
\end{array}\right] \mathrm{N} / \mathrm{m}, } \\
B_{u}= & {\left[\begin{array}{cc}
-1 & 0 \\
0 & -1 \\
1 & 0 \\
0 & 1
\end{array}\right], W_{d}=\left[\begin{array}{ccc}
172000 & 0 & 0 \\
0 & 172000 & 0 \\
0 & 0 & -340 \\
0 & 0 & 340
\end{array}\right] . }
\end{aligned}
$$

The HIS output

$$
u=M_{h} \ddot{z}+C_{h} \dot{z}+K_{h} z+M_{h a} \ddot{v}_{a}+C_{h a} \dot{v}_{a}+K_{h a} v_{a},
$$

where $M_{h}, C_{h}$, and $K_{h}$ are the fluid inertia, damping and stiffness matrices, respectively, associated with the passive behavior of the HIS system. $M_{h a}, C_{h a}$, and $K_{h a}$ are, respectively, the fluid inertia, damping and stiffness matrices associated with the hydraulic actuator input. Neglecting the higher-order terms of the actuator input in (18), we have

$$
u=M_{h} \ddot{z}+C_{h} \dot{z}+K_{h} z+K_{h a} v_{a} .
$$

Consequently, we obtain

$$
\left(M-M_{h}\right) \ddot{z}+\left(C-C_{h}\right) \dot{z}+\left(K-K_{h}\right) z=K_{h a} v_{a}+W_{d} f_{d},
$$

where the inertia, damping and stiffness matrices of the combined system are, respectively, $\mathbf{M}=M-M_{h}, \mathbf{C}=C-C_{h}$ and $\mathbf{K}=K-K_{h}$.

The state variables $\left(x_{1}, x_{2}\right):=(z, \dot{z})$ transform the combined system 20 into the form

$$
\dot{x}=A x+B u+W f_{d}, \quad y=C x,
$$

where the input and output variables are, respectively, $u=v_{a}$ and $y=\left[z_{1}, z_{2}, z_{s}, \theta\right]^{\mathrm{T}}$ (see Fig. 1). The matrices are

$$
\begin{aligned}
A & =\left[\begin{array}{cc}
\mathbf{0} & \mathbf{I} \\
-\mathbf{M}^{-1} \mathbf{K} & -\mathbf{M}^{-1} \mathbf{C}
\end{array}\right], \\
B & =\mathbf{M}^{-1} K_{h a}, W=\mathbf{M}^{-1} W_{d}, \\
C & =\left[\begin{array}{cccccccc}
-1 & 0 & 1 & 0 & 0 & 0 & 0 & 0 \\
0 & -1 & 0 & 1 & 0 & 0 & 0 & 0 \\
0 & 0 & 1 / 2 & 1 / 2 & 0 & 0 & 0 & 0 \\
0 & 0 & -1 /(2 l) & 1 /(2 l) & 0 & 0 & 0 & 0
\end{array}\right] .
\end{aligned}
$$

To determine the resonant frequencies and mode shapes of the combined structure, we diagonalized the matrix $\mathbf{M}^{-1} \mathbf{K}$ as $\mathbf{M}^{-1} \mathbf{K}=\Phi \Lambda \Phi^{-1}$ where the modal matrix $\Phi$ is the columnwise concatenation of the mode-shape vectors and the diagonal frequency matrix $\Lambda$ is listing the eigenvalues $\lambda\left(\mathbf{M}^{-1} \mathbf{K}\right)$ [25]. The principal diagonals entry of the damping matrix are $\Phi^{-1} \mathbf{M}^{-1} \mathbf{C} \Phi=2 \zeta \omega_{n}$. The obtained modal frequencies and damping ratios are, respectively, 1.392, 2.476, 11.808, 12.33 $\mathrm{Hz}$ and $0.041,0.1841,1.789,9.0352$.

Fig. 2 (a) shows the responses of the integrated system under harmonic excitation (consider as a disturbance) at zero initial conditions: $(i)$ the HCM with active suspension control using the proposed FS2SM controller, (ii) the HCM with passive HIS, (iii) the conventional HCM (without any suspension modifications). To compute the passive HIS, we set the hydraulic actuator input $v_{a}=0$. We take $\eta=0.25$ and a Butterworth filter $L(s)=\frac{1}{s^{2}+1.4142 s+1}$ to design the FS2SM controller. We observe that the roll angle is greatly attenuated compare to conventional suspension and passive HIS system. Figs. 2(b) shows the actuator volumetric displacement $v_{a}=\int v(t) d t$. We implement a back-information antiwindup concept in which the difference between the saturated and the unsaturated output (control signal) is used to generate a feedback signal to act on the integrator input.

The roll angle spectrum of the system, excited at low frequency $0.5 \mathrm{~Hz}$ and resonant frequencies $1.392 \mathrm{~Hz}$ and 2.476 $\mathrm{Hz}$, are shown in Fig. 3 . From these figures, we observe that the resonant frequencies are shifted further to prevent natural modes of the system to be excited.

Although frequency-shaped sliding control has the advantage of attenuating the effect of high-frequency unmodeled dynamics [19], making the following substitution in the corresponding discontinuous ones: $\operatorname{sgn}(\dot{\sigma}) \Leftrightarrow \operatorname{sat}\left(\frac{\dot{\sigma}}{\varepsilon}\right)$ or $\operatorname{sgn}(\dot{\sigma}) \Leftrightarrow$ $\tanh \left(\frac{\dot{\sigma}}{\varepsilon}\right)$ or $\operatorname{sgn}(\dot{\sigma}) \Leftrightarrow \frac{\dot{\sigma}}{\dot{\sigma} \mid+\varepsilon}$, a continuous control law can be obtained in order to alleviate the chattering phenomena by smoothing out the control discontinuity in a thin boundary layer, $\mathcal{B}_{\varepsilon}=\{\|\dot{\sigma}\| \leq \varepsilon\}$, neighboring the switching surface [6][10],[9]. We substitute the signum nonlinearity by $\operatorname{sat}(\dot{\sigma} / \varepsilon)$ :

$$
v_{R}=-(\varrho+\eta) \operatorname{sat}(\dot{\sigma} / \varepsilon)-\kappa \dot{\sigma},
$$

in which good approximation of $\operatorname{sgn}(\cdot)$ nonlinearity needs the use of small $\varepsilon$.

Outside the boundary layer, the system dynamics are the same as the switching control

$$
v=v_{E}+v_{R}, \quad\|\dot{\sigma}\| \geq \varepsilon .
$$

Inside the boundary layer

$$
\begin{aligned}
\dot{\sigma}(t) & =s L(s) \theta+\ddot{\theta}=\phi(t) \leq \varepsilon \\
\Rightarrow \theta(t) & =\frac{1}{s L(s)+s^{2}} \phi(t),
\end{aligned}
$$

which represents the system dynamics of $\theta(t)$ under $\phi(t)$. Due to the boundary layer interpolation, after transients the roll angle error is bounded by

$\left|e_{\theta}(t)\right|_{\phi}=\left|\int_{0}^{t} f(\tau) \phi(t-\tau) d \tau\right| \leq \varepsilon \int_{0}^{\infty} f(\tau) d \tau=\varepsilon\|f(t)\|_{1}$,

where $f(t)$ is the impulse response of $\frac{1}{s L(s)+s^{2}}$ (see Fig. $6(\mathrm{a})$ ).

Fig. 4 illustrates that $v_{R}=-(\varrho+\eta) \operatorname{sat}(\dot{\sigma} / \varepsilon)-\kappa \dot{\sigma}$ is over-calculated during computer simulation because of thin 


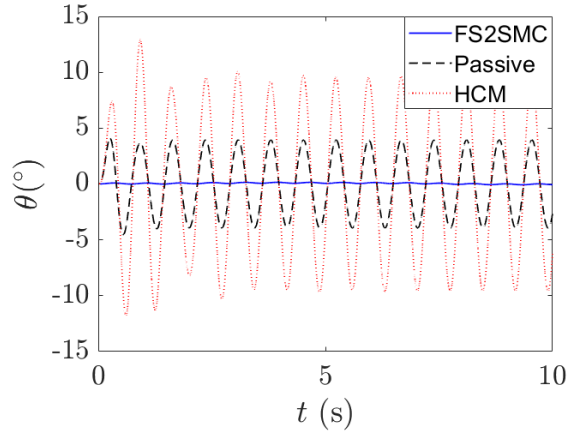

(a) Roll angle

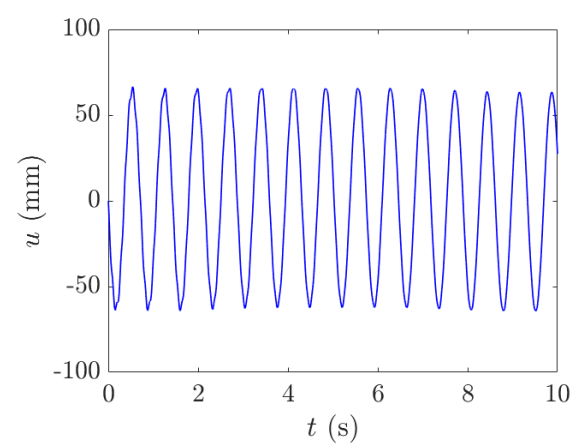

(b) Actuator displacement

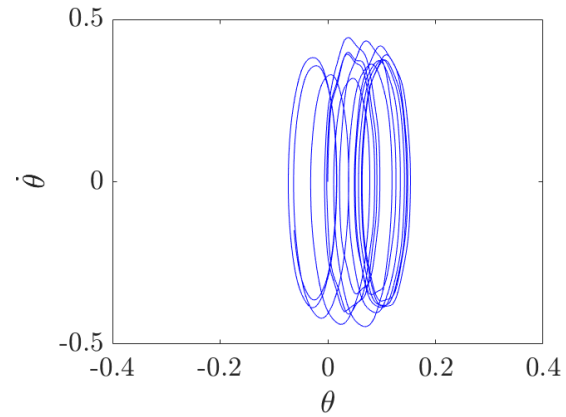

(c) Phase portrait

Fig. 2: Under lateral acceleration $3 \sin (1.392 \times 2 \pi t) \mathrm{m} / \mathrm{s}^{2}$.

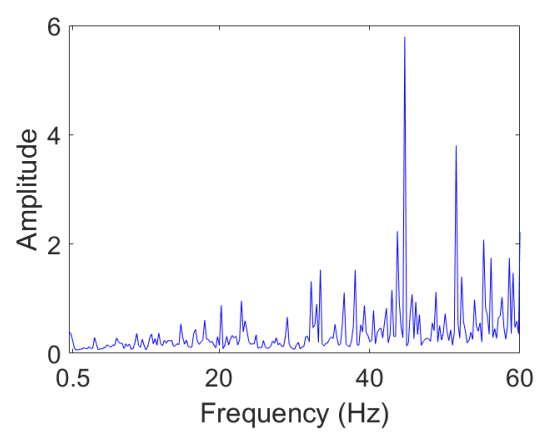

(a) $3 \sin (0.5 \times 2 \pi t) \mathrm{m} / \mathrm{s}^{2}$

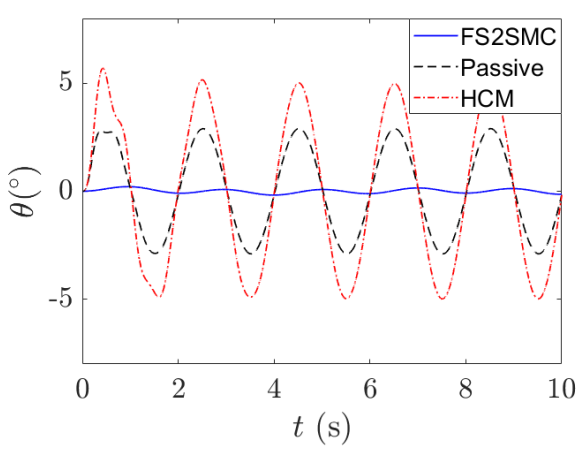

(a) Roll angle

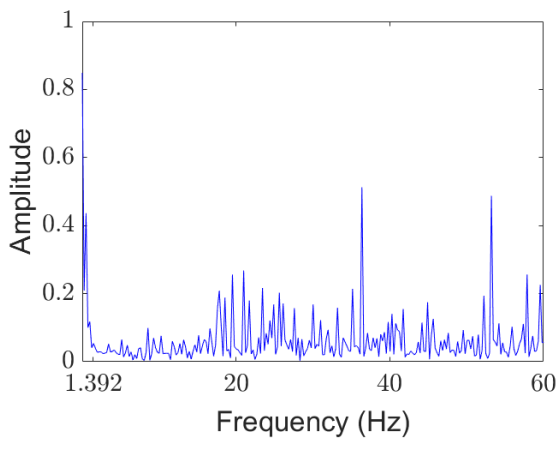

(b) $3 \sin (1.392 \times 2 \pi t) \mathrm{m} / \mathrm{s}^{2}$

Fig. 3: Roll angle spectrum.



(b) Actuator velocity



(c) $3 \sin (2.476 \times 2 \pi t) \mathrm{m} / \mathrm{s}^{2}$

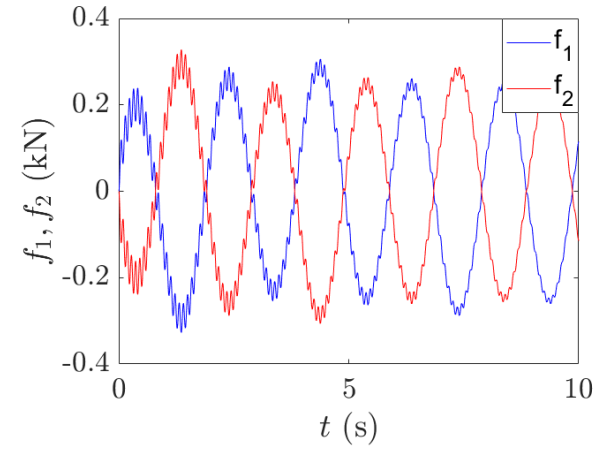

(c) Actuator force

Fig. 4: Responses to $3 \sin (0.5 \times 2 \pi t) \mathrm{m} / \mathrm{s}^{2}$ together with the thin boundary $\left(\varepsilon=\frac{0.05^{\circ}}{\|f(t)\|_{1}}, \kappa=5\right) ; \eta=0.25$.

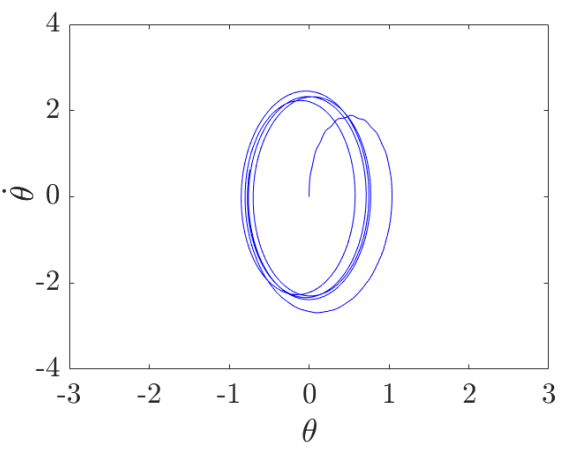

(a) Phase portrait

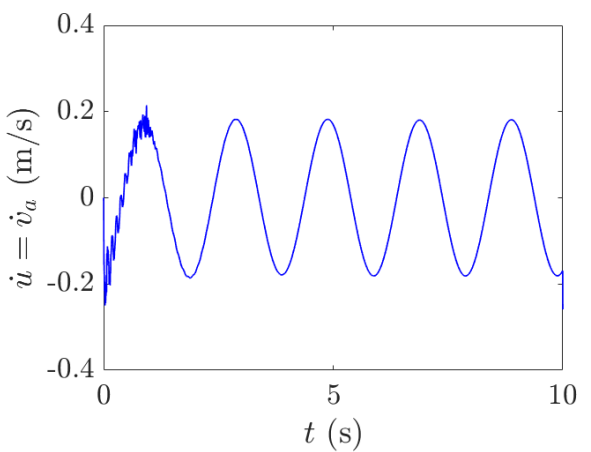

(b) Actuator velocity



(c) Actuator force

Fig. 5: Responses due to a thick boundary $\left(\varepsilon=\frac{0.5^{\circ}}{\|f(t)\|_{1}}, \kappa=0.5\right)$ under lateral acceleration $3 \sin (0.5 \times 2 \pi t) \mathrm{m} / \mathrm{s}^{2} ; \eta=0.25$. 


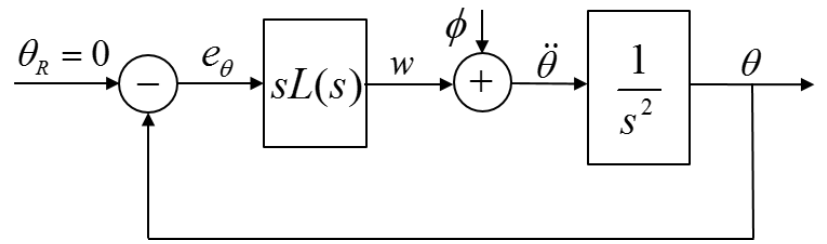

(a)



(b)

Fig. 6: Block diagrams: (a) Feedback system defined by FS2SM, and (b) its LFT representation. $L(s)=\frac{1}{s^{2}+1.4142 s+1}$ but extensions to more orders with an equal number of poles and zeros (or less zeros) can also be made.

boundary layer $\varepsilon=\frac{0.05^{\circ}}{\|f(t)\|_{1}}, \kappa=5$ and $\eta=0.25$. Increasing the boundary layer thickness $\varepsilon=\frac{0.5^{\circ}}{\|f(t)\|_{1}}, \kappa=0.5, \eta=0.25$, we can achieve much smooth responses in term of chattering amplitude attenuation as shown in Fig. 5

According to [4],[8], asymptotic exponential stability is not assured but ultimate boundedness of trajectories to within an $\varepsilon, \kappa$-dependent neighborhood of the origin is guaranteed as shown in Figs. 2(c) and 5(a). Figs. 2(a) and (c) show that the peak error due to a thin boundary layer, $\varepsilon=\frac{0.05^{\circ}}{\|f(t)\|_{1}}, \kappa=5$ ):

$$
\left|e_{\theta}(t)\right|_{\phi}=|-\theta(t)|_{\phi}=\varepsilon\|f(t)\|_{1}<0.2^{\circ},
$$

results in an elliptical trajectories with major and minor axes $0.4^{\circ}$ and $0.04^{\circ}$, respectively. In Fig. 5. a), ultimately the phase portrait is an enclosed ellipse with major and minor axes $2.2^{\circ}$ and $0.7^{\circ}$, respectively, together with the thick boundary $(\varepsilon=$ $\left.\frac{0.5^{\circ}}{\|f(t)\|_{1}}, \kappa=0.5\right)$ for $\dot{\sigma}$ around zero.

We see that 24) is equivalent to a feedback control problem as shown in Fig. 6 a) and it can also be rearranged in linear fractional transformation (LFT) form (see Fig. 6(b)) by

$$
\left[\begin{array}{c}
\theta \\
e_{\theta}
\end{array}\right]=\overbrace{\left[\begin{array}{ccc}
\frac{1}{s^{2}} & 0 & \frac{1}{s^{2}} \\
-\frac{1}{s^{2}} & 1 & -\frac{1}{s^{2}}
\end{array}\right]}^{G(s)}\left[\begin{array}{c}
\phi \\
\theta_{R} \\
w
\end{array}\right],
$$

where $G(s)$ is the generalized plant and $w=s L(s) e_{\theta}$. Hence, state space LQG, $\mathcal{H}_{\infty}$ techniques can also be employed to design the sliding surface.

\section{CONCLUSION}

We have proposed a frequency-shaped 2-sliding mode controller by incorporating a second-order Butterworth function into the sliding surface (identical to a feedback control problem) to shape the frequency characteristics of the equivalent dynamics. We have taken the sliding surface as a manifold modeled by a dynamic linear operator to suppress frequency components of the sliding mode response in a designated frequency band. The system's closed loop performances demonstrate that due to the inclusion of a filter in the control schedule not only reduce the roll angle but also its spectrum can be shaped which makes the active HIS system is potentially useful for a ride and handle control improvement of heavy vehicles, driverless cars, and UGVs. The proposed methodology is beneficial for specific applications where the system's natural frequencies and some frequency bandwidth must be avoided to prevent a structural collapse by resonance. The control schedule (exploiting a notch filter) can also be applied to power electronics where a fixed switching frequency is preferred to control or reduce the switching losses.

\section{REFERENCES}

[1] H. Taghavifar and A. Mardani, Off-road Vehicle Dynamics: Analysis, Modelling and Optimization, Springer Int. Pub., Switzerland, 2017.

[2] Q. P. Ha, S. Royel, J. Li, and Y. Li, "Hysteresis modeling of smart structure MR devices using describing functions," IEEE/ASME Transactions on Mechatronics, vol. 21, no. 1, pp. 44-50, 2016.

[3] T. Tran and Q. Ha, "Fast Algorithm for UGV Wheel-Terrain Interaction Analysis, Proc. IEEE Int. Conf. Control Automation Robotics and Vision, pp. 674-680, Vietnam, Dec. 17-20 Dec., 2008.

[4] H. K. Khalil, Nonlinear Systems, 3rd ed., NJ, USA: Prentice Hall, 2002.

[5] K. Furuta and Y. Pan, "Variable structure control with sliding sector," Automatica, vol. 36, pp. 211-228, 2000.

[6] R.DeCarlo, S.Zak, and G.Matthews, "Variable structural control of nonlinear multivariable systems: A tutorial," Proc. IEEE, 76(3): 212-232, 1988.

[7] J. J. E. Slotine and W. Li, Applied Nonlinear Control, Prentice-Hall, Englewood Cliffs, New Jersey, 1991.

[8] C. Edwards and S. K. Spurgeon, Sliding Mode Control: Theory and Application, London: Taylor \& Francis, 1998.

[9] L. Zuo and J. J. E. Slotine, "Robust vibration isolation via frequencyshaped sliding control and modal decomposition," Journal of Sound and Vibration, vol. 285, pp. 1123-1149, 2005.

[10] J. Liu, Sliding Mode Control Using MATLAB, Elsevier Inc, 2017.

[11] A. F. Filippov, Differential equations with discontinuous right-hand side. Math. and Its App., Kluwer Academic Publishers, Dordrecht, 1988.

[12] A. Levant, "Homogeneity approach to high-order sliding mode design," Automatica, vol. 41, no. 5, pp. 823-830, 2005.

[13] A. Levant, "Principles of 2-sliding mode design," Automatica, vol. 43, no. 4, pp. 576-586, 2007.

[14] Y. Shtessel, C. Edwards, L. Fridman and A. Levant, Sliding Mode Control and Observation, Birkhauser Boston Inc, 2014.

[15] S. Ding, A. Levant and S. Li, "Simple homogeneous sliding-mode controller," Automatica, vol. 67, pp. 22-32, 2016.

[16] Q. P. Ha, M. T. Nguyen, J. Li, and N. M. Kwok, "Smart structures with current-driven MR dampers: modelling and second-order sliding mode control," IEEE/ASME Trans. on Mecha., 18(6), pp. 1702-1711, 2013.

[17] A. Pisano and E. Usai, "Sliding mode Control: a survey with applications in math," Mathematics and Comp in Sim, vol. 81, pp. 954-979, 2011.

[18] K. Young, V. Utkin, and U. Ozguner, "A control engineer's guide to sliding mode control," IEEE Tran. Contr. Syst. Tech, 7(3): 328-342, 1999.

[19] K. D. Young and U. Ozguner, "Frequency shaping compensator design for sliding mode," Int. J. of Control, vol. 57, no. 5, pp. 1005-1019, 1993.

[20] J. X. Xu and W. J. Cao, "Synthesized sliding mode control of a singlelink flexible robot," Int. J. of Control, vol. 73, no. 3, pp. 197-209, 2000.

[21] K. C. Cheok, H. X. Hu, and N. K. Loh, "Discrete-time frequency-shaping parametric LQ control with application to active seat suspension control," IEEE Trans. on Industrial Electronics, vol. 36, no. 3, pp. 383-390, 1989.

[22] A. Mehta and B. Bandyopadhay, "Frequency-shaped sliding mode control using output sampled measurements," IEEE Transactions on Industrial Electronics, vol. 56, no. 1, pp. 28-35, 2009.

[23] Q. P. Ha, S. Royel, and C. Balaguer, "Low-energy structures embedded with smart dampers," Energy and Buildings, vol. 177, pp. 375-384, 2018.

[24] A. Tkachev and N. Zhang, "Active Hydraulically Interconnected Suspension Modeling and Simulation," SAE Tech. Paper, 2017-01-1561, 2017

[25] S. Royel and Q. P. Ha, "Frequency shaped sliding mode control of magnetorheological smart structure systems," Proc. IEEE International Conference on Mechatronics, pp. 117-122, Australia, Feb. 13-15, 2017.

[26] G. Bartolini, A. Ferrara, and E. Usai, "Chattering avoidance by secondOrder sliding mode control,” IEEE Trans. Aut. Con., 43(2):241-246, 1998. 